\title{
Pick-ya actin - a method to purify actin isoforms with bespoke key post-translational modifications
}

\author{
Tomoyuki Hatano*,‡, Lavanya Sivashanmugam*, Andrejus Suchenko, Hamdi Hussain and \\ Mohan K. Balasubramanian ${ }^{\ddagger}$
}

\begin{abstract}
Actin is one of the most abundant eukaryotic cytoskeletal polymerforming proteins, which, in the filamentous form, regulates a number of physiological processes, ranging from cell division and migration to development and tissue function. Actins have different posttranslational modifications (PTMs) in different organisms, including methionine, alanine, aspartate and glutamate $\mathrm{N}$-acetylation, $\mathrm{N}$-arginylation and the methylation of the histidine at residue 73 (His-73), with different organisms displaying a distinct signature of PTMs. Currently, methods are not available to produce actin isoforms with an organism-specific PTM profile. Here, we report the Pick-ya actin method, a method to express actin isoforms from any eukaryote with its own key characteristic PTM pattern. We achieve this using a synthetic biology strategy in a yeast strain that expresses, one, actin isoforms with the desired $\mathrm{N}$-end via ubiquitin fusion and, two, mammalian enzymes that promote acetylation and methylation. Pick-ya actin should greatly facilitate biochemical, structural and physiological studies of the actin cytoskeleton and its PTMs.
\end{abstract}

KEY WORDS: Pichia pastoris, Actin cytoskeleton, Post-translational modification, Recombinant actin

\section{INTRODUCTION}

Actin is a highly conserved eukaryotic cytoskeletal protein, which polymerizes into double-stranded, dynamic polar filaments that exhibit continuous polymerization and depolymerization. A variety of eukaryotes have evolved diverged actin isoforms and orthologues, which assemble into a variety of molecular machineries executing mechanical tasks, including cell division and cell migration. How actins undergo defined molecular structural changes facilitating polymerization and depolymerization, and how they carry out distinct functions at different cellular locations and in different organisms and tissues is not well understood. The understanding of actin function in different organisms and tissues is further complicated by the vast array of PTMs, which differ among different organisms. Some of the known modifications of actin include: (1) methionine $\mathrm{N}$-acetylation in yeast; (2) alanine $\mathrm{N}$-acetylation in Arabidopsis thaliana (Bienvenut et al., 2012);

Centre for Mechanochemical Cell Biology and Division of Biomedical Sciences, Warwick Medical School, University of Warwick, Coventry CV4 7AL, UK.

*These authors contributed equally to this work

${ }^{\ddagger}$ Authors for correspondence (T.Hatano@warwick.ac.uk; M.K.Balasubramanian@warwick.ac.uk)

(iD) M.K.B., 0000-0002-1292-8602

This is an Open Access article distributed under the terms of the Creative Commons Attribution License (https://creativecommons.org/licenses/by/4.0), which permits unrestricted use, distribution and reproduction in any medium provided that the original work is properly attributed.

Received 6 November 2019; Accepted 20 December 2019
(3) aspartate and glutamate $\mathrm{N}$-acetylation in animals and amoeba, mediated by the N-acetyl transferase NAA80 (Drazic et al., 2018; Goris et al., 2018); (4) N-arginylation in mammals (Karakozova et al., 2006); and (5) His-73 methylation in animals and amoeba, mediated by the histidine methyl transferase SETD3 (Kwiatkowski et al., 2018; Wilkinson et al., 2019). Thus, a precise biochemical and biophysical understanding of actin function in various organisms and tissues depends on the ability to produce actin isoforms with organism-specific signature PTMs. The current methods for actin purification either constitutively introduce aspartate and glutamate $\mathrm{N}$-acetylation and His-73 methylation (purification from rabbit or chicken muscle, Sf9-insect cell cultures, Dictyostelium and human platelets) or do not introduce either of the above-mentioned modifications (purification from Saccharomyces cerevisiae and Pichia pastoris) (Hatano et al., 2018; Müller et al., 2013; Noguchi et al., 2007; Ohki et al., 2009).

Given that recombinant actin expressed in $P$. pastoris is neither $\mathrm{N}$-acetylated on aspartate and glutamate residues nor His-73 methylated, we reasoned that further engineering of $P$. pastoris may provide a means to express and purify actin isoforms with distinct organism-specific PTMs. Here, we express NAA80 and SETD3 either singly or together in $P$. pastoris. This, combined with our previously described ubiquitin fusion approach (Hatano et al., 2018), has led to the development of the Pick-ya actin method, which facilitates production of actin isoforms with organismspecific PTM profiles.

\section{RESULTS}

Fig. 1 shows key PTMs observed in actin from mammals, yeast and the plant $A$. thaliana. Mammalian actin can be isolated with one of seven different modifications at the $\mathrm{N}$-terminus, which are mediated by a combination of aminopeptidases, NAA80 acetyl transferase (Drazic et al., 2018; Goris et al., 2018) and ATE1 arginyl-tRNAprotein transferase (Karakozova et al., 2006). Furthermore, human actin is methylated on His-73 by the SETD3 methyl transferase (Kwiatkowski et al., 2018; Wilkinson et al., 2019). A. thaliana actin is N-terminally acetylated at alanine and is only weakly (9\%) methylated on His-74, the equivalent residue to mammalian and yeast His-73 (Wilkinson et al., 2019). Finally, yeast actin is acetylated on the initiator methionine and is not methylated on His73 (Kalhor et al., 1999). We systematically established yeast strains that introduced one or more of the above-mentioned modifications. Towards this end, we cloned human NAA80 acetyl transferase and human SETD3 methyl transferase in $P$. pastoris expression plasmids, which are as described in the subsequent sections.

\section{Expression in $\boldsymbol{P}$. pastoris and purification of $\mathbf{N}$-acetylated human $\beta$ and $\gamma$ actin}

Human $\beta$ - and $\gamma$-actin expressed in $P$. pastoris are not processed at the N-terminus, as a result methionine is retained at the N-terminus 


\begin{tabular}{|c|c|c|c|c|c|}
\hline \multirow{2}{*}{ Species } & \multirow{2}{*}{ Proteins } & \multirow{2}{*}{ Uniprot \# } & \multicolumn{2}{|r|}{ N-terminus } & \multirow{2}{*}{$\begin{array}{c}\text { 73th His } \\
\text { methylation }\end{array}$} \\
\hline & & & PTM & AA seq & \\
\hline \multirow{7}{*}{ H. sapiens } & beta cvtonlasmic & ACTB HUMAN & Ac- & DDDIAALVVD... & + \\
\hline & Neta cytupasmili & RUID_RIVIFIV & Arg- & DDDIAALVVD... & + \\
\hline & gamma cytoplasmic & ACTG_HUMAN & Ac- & EEEIAALVID... & + \\
\hline & alpha skeletal muscle & ACTS_HUMAN & Ac- & DEDETTALVCD... & + \\
\hline & alpha smooth muscle & ACTA_HUMAN & Ac- & EEEDTTALVCD... & + \\
\hline & gamma smooth muscle & ACTH_HUMA & Ac- & EEETTALVCD... & + \\
\hline & alpha cardiac muscle & ACTC_HUMAN & Ac- & DDEETTALVCD... & + \\
\hline \multirow[t]{2}{*}{ S. cerevisiae } & Act1p & ACT_YEAST & Ac- & MDSEVAALVI... & - \\
\hline & Actin-1 & ACT1_ARATH & Ac- & ADGEDIQPLVC... & $-1+$ \\
\hline \multirow[t]{2}{*}{ A. thaliana } & Actin-2 & ACT2_ARATH & Ac- & AEADDIQPIVC... & $-/+$ \\
\hline & Actin-7 & ACT7_ARATH & Ac- & ADGEDIQPLVC... & $-1+$ \\
\hline
\end{tabular}

Fig. 1. Post-translational modification of actin. Divergent post-translational modifications on actin. Shown are $\mathrm{N}$-terminal peptide sequences of nine actins (six from $H$. sapiens, one from S. cerevisiae, and three from $A$. thaliana), and the observed modifications at the $\mathrm{N}$-terminus. The right column summarizes whether His-73 methylation is observed in these organisms and is acetylated by native acetyl transferases (Hatano et al., 2018). The canonical $\mathrm{N}$-acetylation occurs on an aspartate residue on $\beta$-actin and a glutamate residue on $\gamma$-actin. To facilitate generation of $\beta$ - and $\gamma$-actin with aspartate or glutamate at the N-terminus, we expressed a DNA molecule (Fig. 2A) encoding a translational fusion of ubiquitin with the actin gene lacking the initiator methionine followed by sequences encoding human thymosin $\beta 4$ (Tß4) and the eight consecutive histidine amino acid residues (8His), which facilitates one-step affinity purification using nickelNTA resin (Hatano et al., 2018; Huang et al., 2016; Kijima et al., 2016; Noguchi et al., 2007). In this strain, we introduced an integrative plasmid vector backbone or a plasmid expressing human NAA80, which promotes actin $\mathrm{N}$-acetylation. The translational fusion was purified as described previously using an affinitybinding approach (Hatano et al., 2018). When the four purified actin fusions were subjected to mass spectrometry (MS), we found that the majority of the $\mathrm{N}$-termini in $\beta$ actin contained an aspartate residue, whereas a large number of acetylated aspartate (Ac-Asp)containing peptides were detected when NAA80 was co-expressed in these cells (Fig. 2B,D; Fig. S1A). Similarly, the majority of $\gamma-$ actin contained a glutamate residue at the N-terminus in control cells, whereas it contained acetylated glutamate (Ac-Glu) at the $\mathrm{N}$-terminus when NAA80 was co-expressed (Fig. 2C,E). Evaluation of the number of non-acetylated versus acetylated N-terminal peptides from $\beta$ - and $\gamma$-actin clearly demonstrated that NAA80 expression strongly promotes the $\mathrm{N}$-terminal acetylation of $\beta$ - and $\gamma$-actin. Together, we concluded that expression of NAA80 is sufficient to introduce $\mathrm{N}$-acetylation of human $\beta$ and $\gamma$ actin expressed in $P$. pastoris.

\section{Expression in P. pastoris and purification of His-73 methylated human $\beta$ - and $\gamma$-actin}

We next established a system to prepare His-73-methylated human $\beta$ - and $\gamma$-actin. Recent work has shown that the mammalian SETD3 methyl-transferase methylates His-73 of human actins, using $S$-adenosyl methionine as a methyl group donor (Kwiatkowski et al., 2018; Wilkinson et al., 2019; Fig. 3A). To promote actin His73 methylation, we co-expressed the mammalian SETD3 and $\beta$ - or $\gamma$-actin in $P$. pastoris cells. Again, methylated peptides covering His-73 were detected in cells expressing SETD3, but not in cells without SETD3 (Fig. 3B-E; Fig. S1B). Both, $\beta$ - and $\gamma$-actin were methylated on His-73 (Fig. 3B,C). Quantification of the relative intensity of methylated versus non-methylated peptides revealed a clear correlation between expression of SETD3 and methylation of
His-73 on $\beta$ - and $\gamma$-actin expressed in P. pastoris (Fig. 3D,E). These experiments established that human $\beta$ - and $\gamma$-actins can be efficiently methylated by SETD3 in P. pastoris.

\section{Expression in P. pastoris and purification of $\mathbf{N}$-acetylated and His-73 methylated human $\beta$-actin, and arginylated and His-73 methylated $\gamma$-actin}

Next, we sought to purify dually modified human $\beta$-actin with $\mathrm{N}$-acetylation and His-73 methylation. Towards this goal, we employed a ubiquitin-fusion strategy as described in Fig. 2A. In $P$. pastoris cells expressing the Ub-D-actin-T $\beta 4-8$ His fusion, we expressed NAA80 and SETD3. Actin was purified sequentially by nickel affinity chromatography, release from $\mathrm{T} \beta 4$ and 8 His by chymotrypsin treatment, and a cycle of polymerization and depolymerization (see Materials and Methods for full details of the purification). Fig. 4A shows results from a size exclusion column, in which monomeric actin was obtained in the peak fractions. Mass spectrometric analysis of the purified actin confirmed that it was both $\mathrm{N}$-acetylated and His-73 methylated (Fig. S2), as described in Figs 2 and 3.

Next, we tested whether the purified N-acetylated and His-73 methylated human $\beta$-actin was capable of undergoing polymerization. Towards this goal, we incubated the purified human actin with a trace amount of $S$. cerevisiae actin that was fluorescently labelled by conjugation of Alexa Fluor 488 C5maleimide to Cys-374 and compared the elongation rate with human platelet actin (commercially available from Cytoskeleton, Inc.). In this experiment, we found that the dually modified recombinant actin was capable of polymerization with comparable elongation rate $(\sim 3.5$ subunits $/ \mathrm{s} / \mu \mathrm{M})$ to the platelet actin in two independent experiments (Fig. 4B-D).

While $\mathrm{N}$-acetylation is one key modification of the N-terminus of actin, a mutually exclusive modification termed arginylation also occurs on human $\beta$-actin. We have shown that the ubiquitin fusion strategy can be used to express and purify arginylated human $\beta$-actin from P. pastoris (Hatano et al., 2018). To develop a full actin toolkit, we sought to express and purify arginylated and methylated actin. To this end, we co-expressed the Ub-R-actin-T $\beta 4-8$ His fusion and SETD3 in P. pastoris. We were able to purify arginylated and His73 methylated actin at levels comparable to N-acetylated and His-73 methylated actin (Fig. 4E; Fig. S3).

Collectively, these experiments show that the system we have developed can allow a mix and match strategy to purify actin as (1) unmodified, (2) N-acetylated and unmethylated, (3) 
A Human beta/gamma actin with $\mathrm{N}$-acetylation

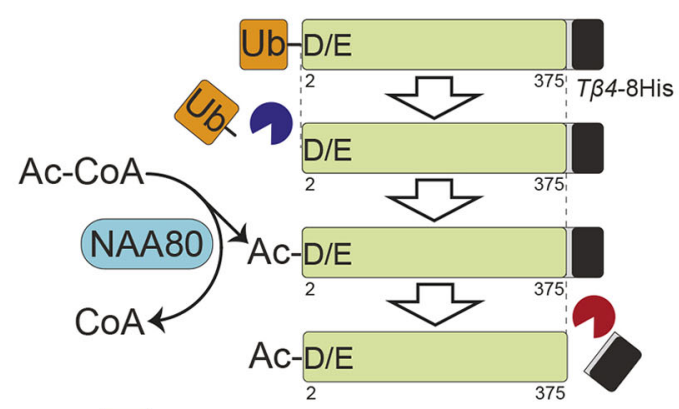

Ub-Ubiquitin tag

Ub protease

$>$ Chymotrypsin

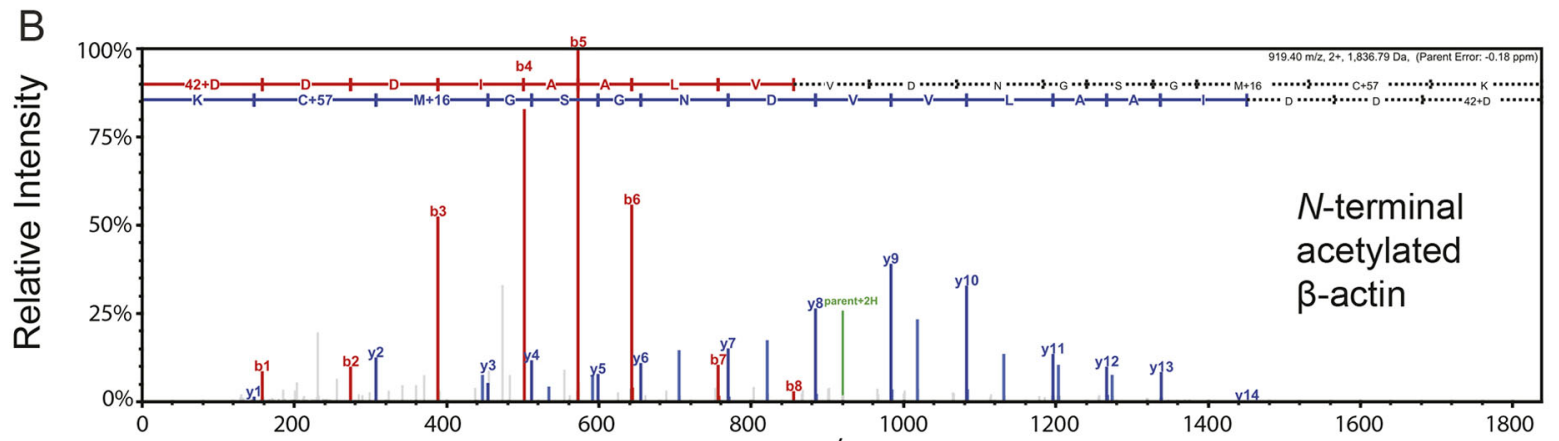

C
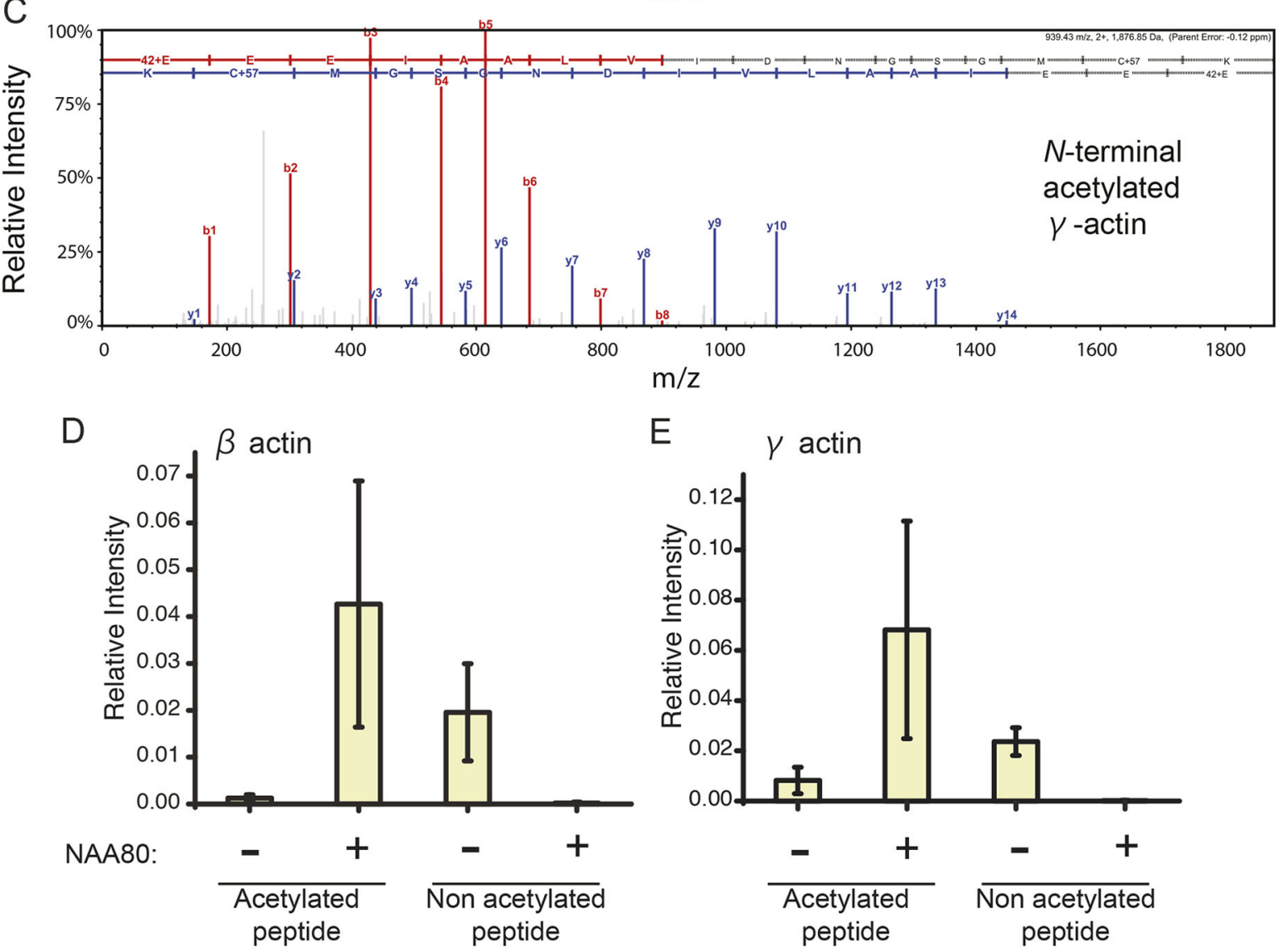

Fig. 2. See next page for legend. 
Fig. 2. $\mathbf{N}$-acetylation of recombinant human $\boldsymbol{\beta}$ - and $\gamma$-actin. (A) Schematic representation of purification of synthetically $\mathrm{N}$-acetylated human $\beta$ - and $\gamma$-actin. A ubiquitin (Ub) moiety was fused at the $\mathrm{N}$-terminus of actin lacking the first methionine residue. The Ub tag is removed by the endogenous Ub protease and the exposed $\mathrm{N}$-terminal aspartate residue in $\beta$-actin or glutamate residue in $\gamma$ actin is acetylated by the NAA80 enzyme. The $\mathrm{C}$-terminal tag is then removed by chymotrypsin treatment to liberate modified full-length actin. $(B, C) M S$ spectra of the $N$-terminal peptide derived from recombinant $\beta$-actin $(B)$ and $\gamma$-actin $(C)$. $(D, E)$ Label-free quantification of acetylated or unmodified $N$-terminal peptide in $\beta$ - and $\gamma$-actin. The bar graphs shows mean \pm s.e.m. from three independent experiments. The relative intensity of $\mathrm{N}$-acetylated peptide in control sample and the sample expressing NAA80 is shown; (D) $\beta$ actin, (E) $\gamma$-actin.

$\mathrm{N}$-acetylated and methylated, (4) arginylated and unmethylated, and (5) arginylated and unmethylated forms.

\section{Expression in $\boldsymbol{P}$. pastoris and purification of alanine $\mathbf{N}$-acetylated and His-73 methylated A. thaliana actin}

We next wanted to extend the utility of the approach we have developed to purify actin isoforms. We were in particular interested to use our system to express plant actin isoforms since they have unique profiles of PTMs (Fig. 1). Actins from the model plant A. thaliana have been shown, through proteomic analysis, to be $\mathrm{N}$-acetylated on alanine (rather than aspartate or gluatamate, as seen in mammalian actins), and methylation of His-74 is only found in $\sim 9 \%$ of the cellular pool of actin (Bienvenut et al., 2012; Wilkinson et al., 2019). We reasoned that the $P$. pastoris expression system might offer a better avenue to producing plant actins due to our ability to control each of the modifications. We generated a fusion of the A. thaliana Actin-2 (Act2) gene, in which we again used the ubiquitin fusion strategy, to allow exposure of the alanine residue at position 2 at the N-terminus (Fig. 5A). A Ub-A-actin-T $\beta 4-8 \mathrm{His}$ fusion was expressed in P. pastoris. We found, through MS, that the exposed $\mathrm{N}$-terminal alanine residue was acetylated by a native acetyl transferase (Fig. 5B; Fig. S1C). This observation established that $A$. thaliana actin can be expressed in $P$. pastoris in a $\mathrm{N}$-acetylated form for biochemical studies.

Since $\sim 9 \%$ of $A$. thaliana actin is methylated on His-74 (corresponding to His-73 in animal actin), we desired to establish a system to purify His-74 methylated actin (Wilkinson et al., 2019). To this end, we expressed SETD3 in $P$. pastoris expressing Ub-Aactin-T $\beta 4-8$ His. MS confirmed that the A. thaliana actin was methylated on His-74 (Fig. 5C,D; Fig. S1C). Comparison of the relative intensity profiles of methylated and non-methylated peptides revealed a clear correlation between SETD3 expression and His-74 methylation (Fig. 5D). This work established that $A$. thaliana actin can be expressed in an alanine $\mathrm{N}$-acetylated form with or without His-74 methylation.

Finally, we tested whether the purified alanine $\mathrm{N}$-acetylated A. thaliana actin was capable of polymerization in vitro. We performed a similar experiment to that with the human $\beta$-actin described in Fig. 4B-D, and found that A. thaliana actin polymerized at $\sim 3-4$ monomers $/ \mathrm{s} / \mu \mathrm{M}$ in two independent experiments (Fig. 5E). This finding established that $A$. thaliana actin purified from $P$. pastoris was functional in vitro (Fig. S4).

\section{DISCUSSION}

In this study, we have substantially improved upon our previous work (Hatano et al., 2018) on expression and purification of heterologous actin isoforms in $P$. pastoris. Importantly, we have developed approaches to precisely control the N-acetylation and His-73 methylation status of expressed heterologous actin forms. As previously reported, the yield of the actin was $\sim 0.5 \mathrm{mg}$ from 11 yeast culture in our set up using standard glass flasks for the yeast culture (Hatano et al., 2018). The yield could be further improved by using high-aeration culture, which can achieve highdensity cultivation of the yeast. We see two major advantages for this system we describe, which we term as Pick-ya actin.

First, Pick-ya actin will facilitate biochemical and structural studies of actin isoforms, and their modifications. We imagine that any laboratory investigating the role of each of the modifications of mammalian $\beta$ - and $\gamma$-actin on its structural, biochemical and biophysical properties could now work with pure actin isoforms with the desired modifications. Actin without modifications can be readily isolated without the need for cell lines lacking NAA80 or SETD3, and actins with modifications can be purified upon co-expression of the appropriate enzymes.

Second, and perhaps more importantly, Pick-ya actin could help experimenters understand the diversity of actin function in a variety of different organisms with different profiles of $\mathrm{N}$-acetylation and His-73 methylation. In this regard, Pick-ya actin may be preferable to the Sf9 insect cell culture-based approach. This is due to the fact that wild-type $P$. pastoris cells do not appreciably posttranslationally modify heterologous actins either by $\mathrm{N}$-acetylation or His-73 methylation, whereas Sf9 cells do. The ability to express unmodified actin, yet promote $\mathrm{N}$-acetylation or His-73 methylation by expression of appropriate enzymes, as and when required, offers the potential to generate the correct actin isoform with the appropriate PTMs for biochemical, biophysical and structural studies. For example, study of $A$. thaliana actin in vitro could employ a mixture of alanine acetylated actin with a fraction $(\sim 10 \%)$ that is His-73 methylated and the rest unmethylated. In this regard, we believe Pick-ya actin will facilitate the study of actins from a large variety of eukaryotes, including plants. Given that methods are not available to purify actin isoforms from native sources presently, it will be important to compare the performance of Pick-ya actin isoforms with actin isoforms purified from native sources at such a time when such methods are developed.

A simple flow chart for actin expression and purification requires establishing (1) whether actin isolated from the source contains $\mathrm{N}$-methionine, alanine, glutamate or aspartate $\mathrm{N}$-acetylation, (2) whether His-73 is methylated and (3) whether the C-terminal amino acid is a phenylalanine. Based on the answers to these questions, expression of appropriate ubiquitin-actin-T $\beta 4-8 \mathrm{His}$ fusion proteins with or without NAA80 and SETD3 and subsequent purification should provide pure actin isoforms with appropriate modifications.

\section{MATERIALS AND METHODS \\ Plasmids and strains}

Plasmids and strains used in this study are listed in Tables S1 and S2, respectively.

\section{pPICZc-actin-thymosin $\boldsymbol{\beta} \mathbf{4 - 8 H i s}$}

Actin-coding sequences codon-optimized for expression in P. pastoris were synthesized by gBlocks (IDT) and cloned into pPICZc (Invitrogen), which adds, at the C-terminus, an in-frame chymotrypsin cleavage site, linker sequence, thymosin $\beta 4$ and a His tag (Noguchi et al., 2007; Hatano et al., 2018).

\section{N-terminal ubiquitin fusion constructs}

The $5^{\prime}$ portion of the $S$. cerevisiae UBI4 gene encoding ubiquitin repeat 1 (encoding amino acids 1-76) were PCR amplified from genomic DNA isolated from $S$. cerevisiae and cloned at the $5^{\prime}$ end of in-frame actin in pPICZc-actinthymosin $\beta 4-8$ His plasmid linearized by EcoRI using Gibson assembly. The first ATG for actin was removed as part of the cloning. 
A

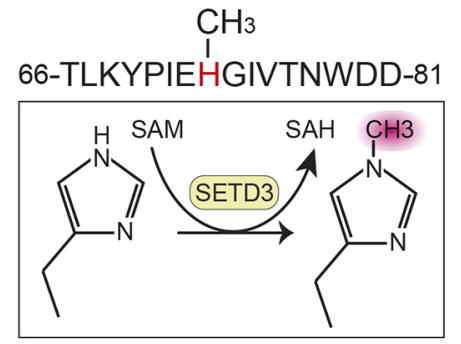

B
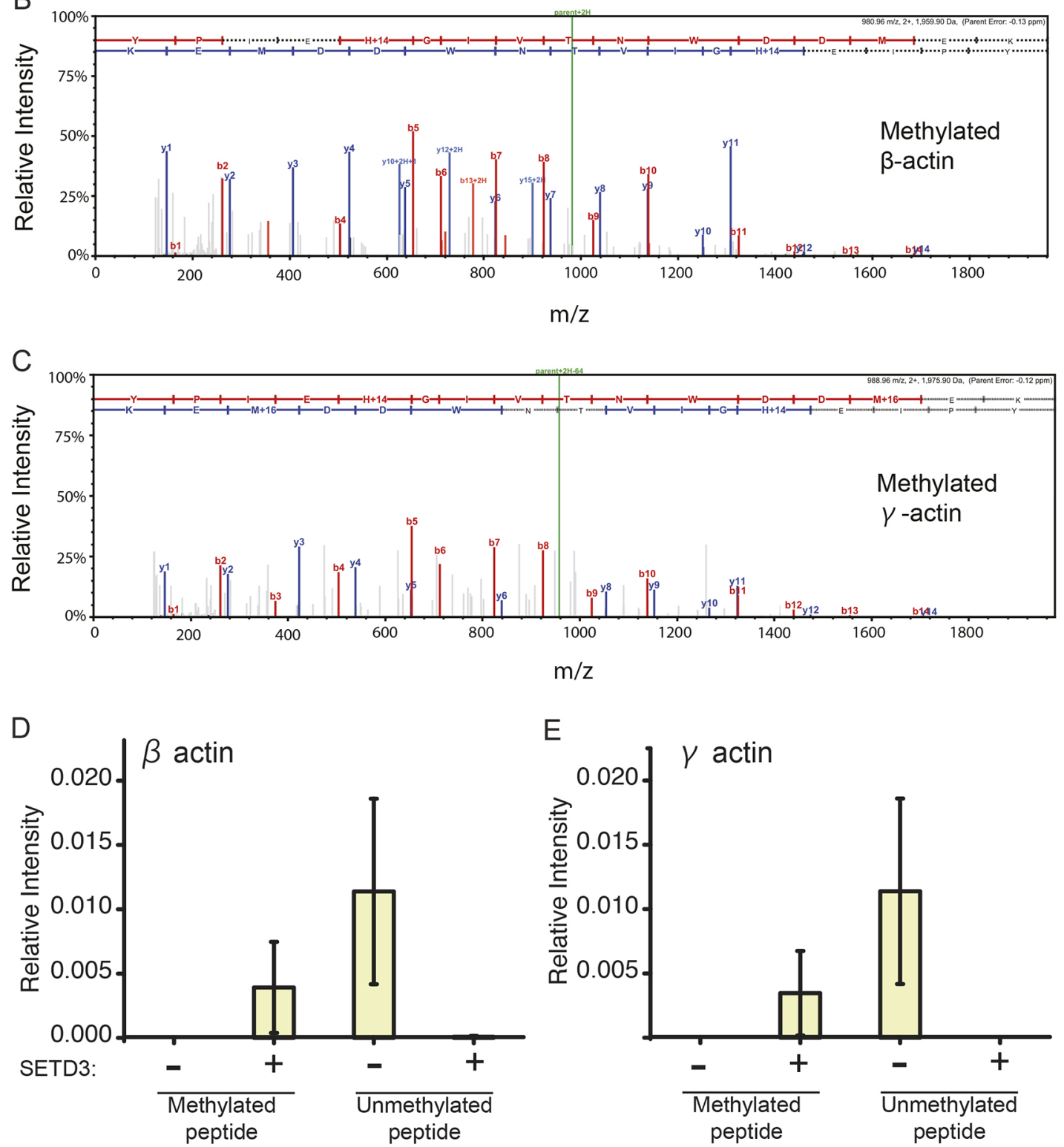

Fig. 3. Methylation of the residue His-73 in human $\beta$ - and $\gamma$-actin. (A) Enzymatic reaction catalysed by the SETD3 methyl transferase. The peptide sequence from human non-muscle actin around the amino acid residues 66-81 are shown. SETD3 recognizes the peptide surrounding His-73 in actin and transfers a methyl group from S-adenosyl methionine (SAM) to the histidine residue to generate methylated actin and S-adenosyl homocysteine (SAH). (B,C) MS spectra of the peptide from recombinant actin carrying methylation at His-73. (B) $\beta$ actin, (C) $\gamma$-actin. (D,E) Label-free quantification of methylated or unmodified His-73 Peptide in $\beta$ - and $\gamma$-actin. The bar graph shows mean \pm s.e.m. from three independent experiments. The relative intensity of methylated peptide in control sample and the sample expressing SETD3 is shown; (D) $\beta$ actin, (E) $\gamma$-actin.

\section{NAA80 and SETD3 genes}

NAA80 and Myc-SETD3 genes codon-optimized for expression in P. pastoris were synthesized by gBlocks (IDT) and cloned into $\mathrm{pIB} 2(P G A P$ promoter) or pIB4 (PAOX1 promoter) $P$. pastoris expression vectors by Gibson assembly.

\section{Dual expression of NAA80 and myc-SETD3}

A PCR-amplified PGAP:NAA80::TAOXI DNA fragment was cloned at the $3^{\prime}$ end of the $N A A 80$ gene. The resulting plasmid lacking the terminator for 
A

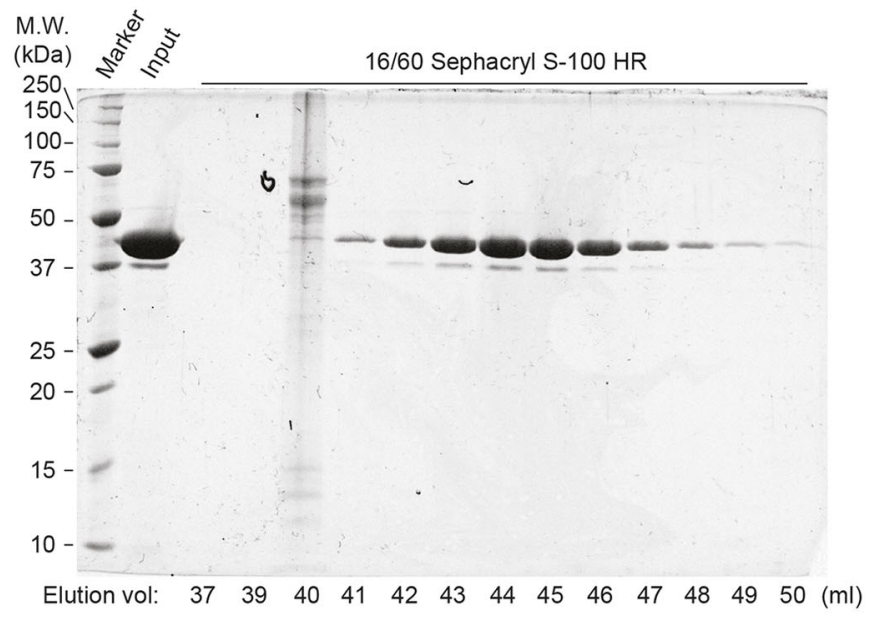

$\mathrm{B}$

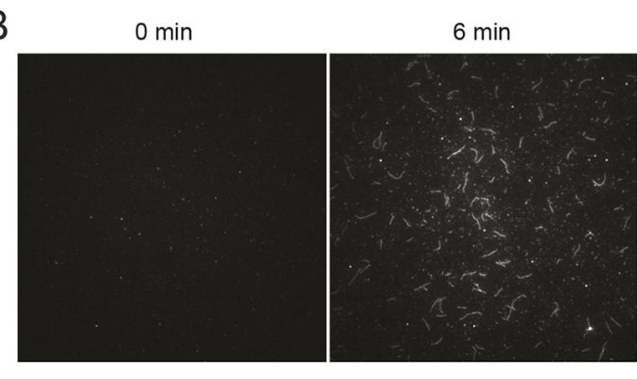

C
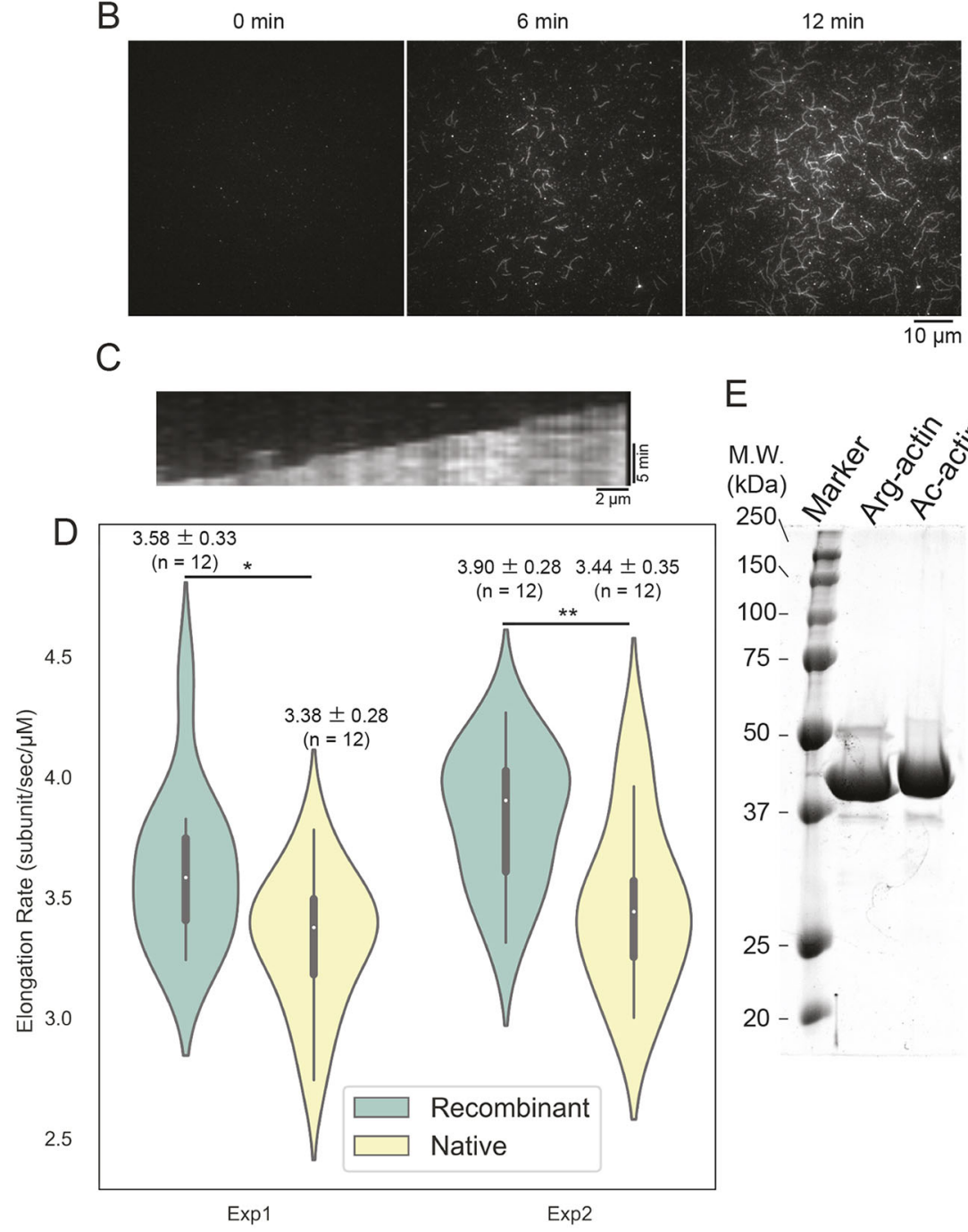

$\overline{10 \mu \mathrm{m}}$

$E$

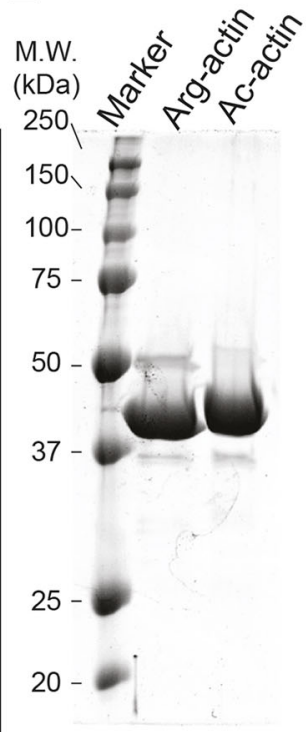

Fig. 4. Purification of dually modified human $\beta$-actin. (A) Size-exclusion chromatography (Sephacryl S-100HR) showing homogenous $\mathrm{G}$-actin purified from $P$. pastoris expressing NAA80 and SETD3. (B) Time-lapse images showing actin polymerization. Actin was visualized by means of incorporation of $20 \%$ S. cerevisiae (Sc)Act 1 conjugated to Alexa Fluor 488. F-actin was tethered on the coverslip surface through the use of $0.5 \%$ ScAct 1 with biotin-PEG2. (C) Kymograph (right) showing elongation of a single actin filament. (D) Elongation rate of recombinant and native (human platelet) actin. Elongation rate (subunit/ second $/ \mu \mathrm{M}$ ) at the barbed end of actin was quantified as described in the Materials and Methods. Data from two independent experiments $(n=12)$ for each actin are shown in the figure as a violin plot. Welch's $t$-test showed significant difference between elongation rate of 'Recombinant' and 'Native' actin (exp1: * $P=0.025$; exp2: $\left.{ }^{* *} P=0.0096\right)$. Mean \pm s.d. values are given above each plot. (E) SDS-PAGE gel of purified arginylated $\beta$-actin (Argactin) carrying methylation at His-73. N-acetylated and His73 methylated $\beta$-actin is also shown in this gel. the NAA80 gene was linearized by PstI/SphI digestion and CYC1 terminator was cloned by ligation.

\section{P. pastoris culture}

The composition of the minimal glycerol (MGY) and minimal methanol (MM) growth media, and basic techniques for P. pastoris are described in the Pichia Expression Kit Instruction Manual (https://www.thermofisher. com/order/catalog/product/V19020).

\section{P. pastoris transformation}

Plasmid DNA was linearized, and yeast cells were transformed by using the lithium chloride method or electroporation. Transformants with the pPICZc plasmid were selected on yeast extract peptone dextrose (YPD) solid medium containing $100 \mathrm{mg} / \mathrm{l}$ Zeocin (Gibco, \#R25001). For transformation of cells with pIB2 or pIB4 plasmids, his 4 cells were used as the host, and transformants were selected on MGY. Single colonies were picked and cultured on YPD solid medium, and the transformants were re-selected to select stable clones. 
A

Ub-Ubiquitin tag

\section{A. thaliana actin}

Ub protease

Chymotrypsin $\mathrm{Ub}-\mathrm{A}$

\section{with $\mathrm{N}$-acetylation}
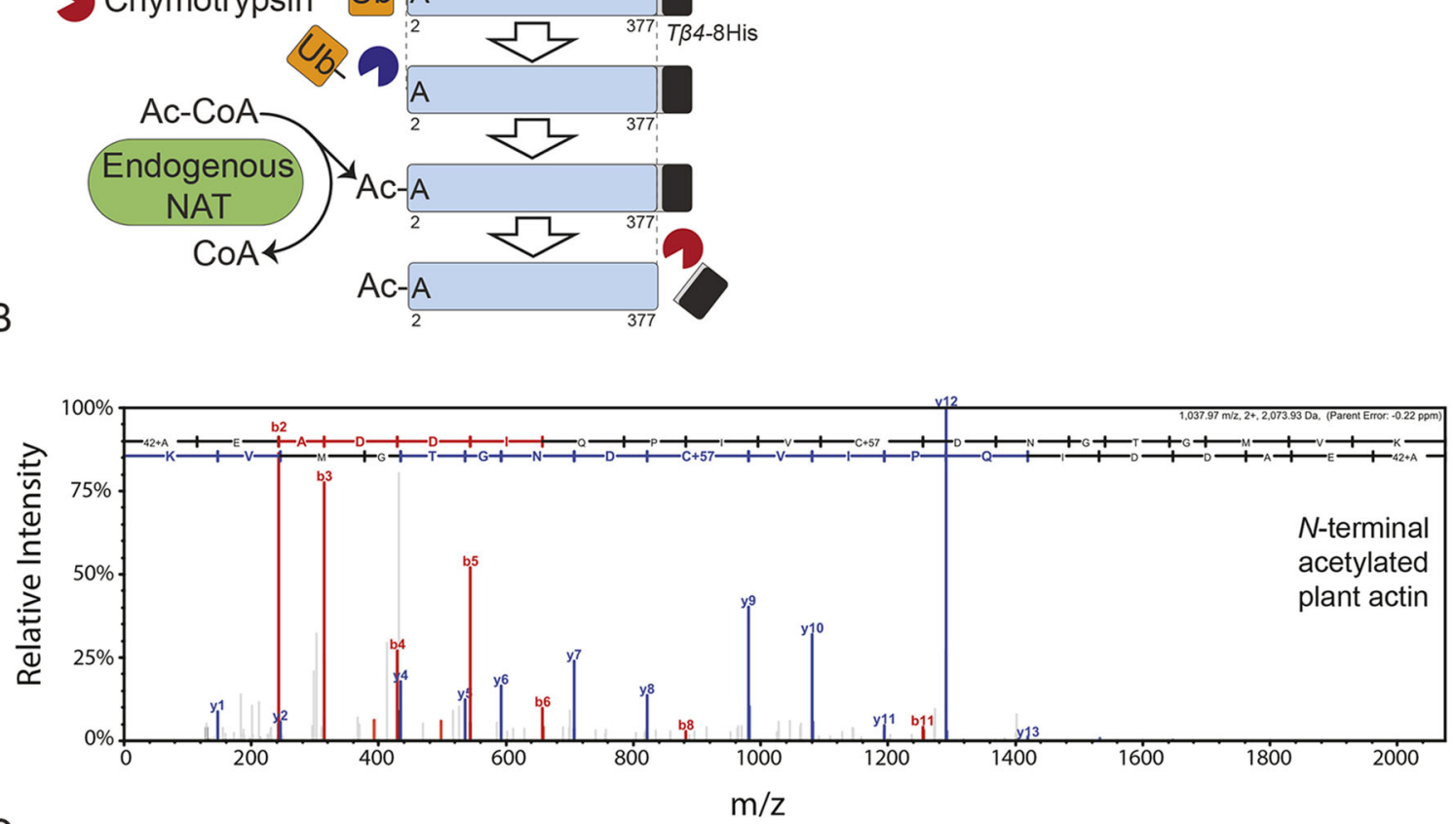

C
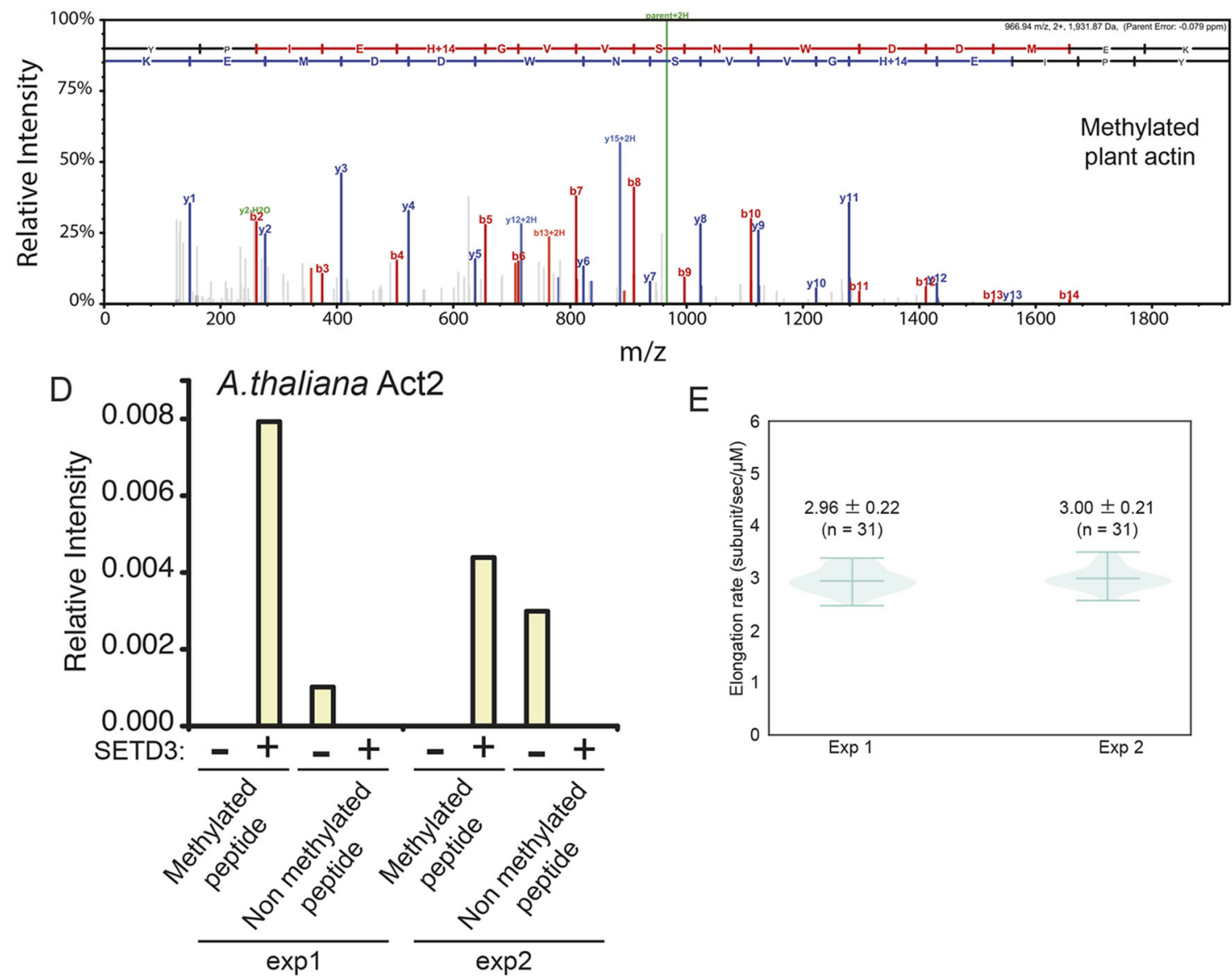

E

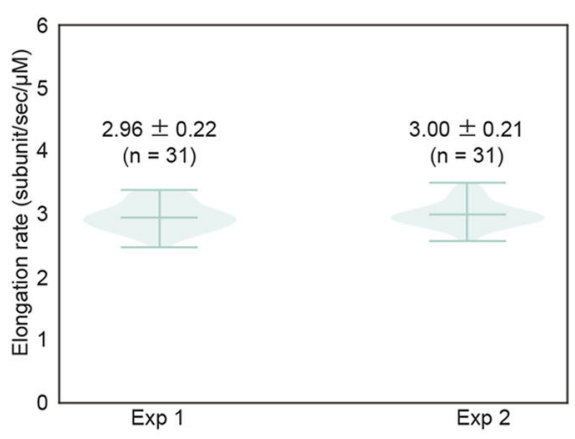

Fig. 5. See next page for legend. 
Fig. 5. A. thaliana Actin-2 with appropriate PTMs. (A) Schematic representation of purification of $A$. thaliana actin. A Ub moiety was fused at the $\mathrm{N}$-terminus of actin lacking the first Met. The Ub tag is removed by the endogenous $\mathrm{Ub}$ protease and the exposed $\mathrm{N}$-terminal alanine residue is $\mathrm{N}$-acetylated by a native acetyl transferase. The $\mathrm{C}$-terminal tag is then removed by chymotrypsin treatment to liberate modified full-length actin. (B) MS spectra of acetylated peptides in recombinant $A$. thaliana Actin-2. (C) Methylation at His-74 in A. thaliana Actin-2 in the presence of SETD3. (D) Label-free quantification of methylated and unmodified His-73 peptide in $A$. thaliana (At) actin. Two independent experiments (exp1 and exp2) were performed.

The relative intensity of methylated peptide in control sample and the sample expressing SETD3 is shown. (E) Elongation rate of recombinant $A$. thaliana Actin-2. Elongation rate (subunit/second/ $\mu \mathrm{M}$ ) of recombinant $A$. thaliana Actin-2 at the barbed end of actin was quantified as described in Fig. 4D and Materials and Methods. The results of two independent experiments $(n=31)$ are shown in the figure as mean \pm s.d.

\section{Purification of recombinant actin from $\boldsymbol{P}$. pastoris}

Actin was purified as described previously (Hatano et al., 2018) with slight modifications. $P$. pastoris transformants were inoculated into MGY liquid medium and cultured at $30^{\circ} \mathrm{C}$, with shaking at $220 \mathrm{rpm}$. The culture medium was diluted and cells were further cultured at $30^{\circ} \mathrm{C}$, with rotation at $220 \mathrm{rpm}$, until the optical density at $600 \mathrm{~nm}\left(\mathrm{OD}_{600}\right)$ reached $\sim 1-1.5$. Cells were pelleted by centrifugation at $10,628 \mathrm{~g}$ at $25^{\circ} \mathrm{C}$ for $10 \mathrm{~min}$ (Thermo Fisher Scientific, \#F9-6×1000 LEX rotor) and washed once with sterilized water prior to re-suspension into MM medium. Cells were cultured in 21 baffled flasks $\left(500 \mathrm{ml}\right.$ culture each) at $30^{\circ} \mathrm{C}$, with rotation at $220 \mathrm{rpm}$, for 1.5 2 days; $0.5 \%$ methanol was added every $24 \mathrm{~h}$ of culturing. Cells were washed once with water and resuspended in water. The suspension was dripped into liquid nitrogen to freeze and stored at $-80^{\circ} \mathrm{C}$ until use.

Frozen cells were loaded into a grinder tube (maximum $50 \mathrm{~g}$ of sample, \#6801, SPEX ${ }^{\circledR}$ SamplePrep) pre-cooled with dry ice and ground in a Freezer mill (\#6870, SPEX ${ }^{\circledR}$ SamplePrep) in a liquid nitrogen bath. The duration of the grinding was 1 min with 14 cycles per second (cps). The grinding was repeated 40 times at $1 \mathrm{~min}$ intervals. Liquid nitrogen was re-filled every 10 cycles of the grinding. The lysate powder was stored at $-80^{\circ} \mathrm{C}$ until further use. The lysate was resolved in an equal amount of $2 \times$ binding buffer [20 mM imidazole, $20 \mathrm{mM}$ HEPES pH 7.4, $600 \mathrm{mM} \mathrm{NaCl}, 4 \mathrm{mM} \mathrm{MgCl}_{2}$, $2 \mathrm{mM}$ ATP, $2 \times$ concentration of protease inhibitor cocktail (cOmplete, EDTA free \#05056489001, Roche), $1 \mathrm{mM}$ phenylmethylsulfonyl fluoride (PMSF) and $7 \mathrm{mM} \beta$-mercaptoethanol ( $\beta$-ME)]. The lysate was sonicated on ice (10 s with 60\% amplitude, Qsonica Sonicators) until all aggregates were resolved. The lysate was centrifuged at $4{ }^{\circ} \mathrm{C}$ at $3220 \mathrm{~g}$ for $15 \mathrm{~min}$ (Eppendorf \#A-4-81 rotor) to remove intact cells and debris, then further cleared by centrifugation at $4^{\circ} \mathrm{C}$ and 25,658 $\mathrm{g}$ for $1 \mathrm{~h}$ (Thermo Fisher Scientific, \#A23$6 \times 100$ rotor). The supernatant was passed through a $0.22 \mu \mathrm{m}$ filter (Corning \#431097) and incubated with nickel resin (Thermo Fisher Scientific, $\# 88222$ ) at $4^{\circ} \mathrm{C}$ for $1-1.5 \mathrm{~h}$. The resin was pelleted by centrifugation at $4^{\circ} \mathrm{C}$ at $1258 \mathrm{~g}$ for $5 \mathrm{~min}$ (Eppendorf \#A-4-81 rotor) and washed with $25 \mathrm{ml}$ icecold binding buffer [10 mM imidazole, $10 \mathrm{mM}$ HEPES pH 7.4, $300 \mathrm{mM}$ $\mathrm{NaCl}, 2 \mathrm{mM} \mathrm{MgCl} 2,1 \mathrm{mM}$ ATP, protease inhibitor cocktail (cOmplete, EDTA free \#05056489001, Roche), $1 \mathrm{mM}$ PMSF and $7 \mathrm{mM} \beta$-ME]. The resin was loaded into an open column (Bio-Rad, \#731-1550) and washed first with ice-cold $20 \mathrm{ml}$ binding buffer, then with $45 \mathrm{ml}$ ice-cold G-buffer [5 mM HEPES pH 7.4, $0.2 \mathrm{mM} \mathrm{CaCl}, 0.01 \%$ (w/v) $\mathrm{NaN}_{3}, 0.2 \mathrm{mM}$ ATP and $0.5 \mathrm{mM}$ dithiothreitol (DTT)]. The resin was resuspended in $6 \mathrm{ml}$ icecold G-buffer containing $5 \mu \mathrm{g} / \mathrm{ml}$ TLCK-treated chymotrypsin (Sigma, \#C3142) and incubated overnight at $4^{\circ} \mathrm{C}$. The chymotrypsin was inactivated by addition of PMSF to $1 \mathrm{mM}$ and incubated for $30 \mathrm{~min}$ on ice. The eluate was then collected into a tube. Actin retained on the resin was eluted with G-buffer and all the elution fractions were combined. The eluate was concentrated using a $30 \mathrm{kDa}$ cut-off membrane (Sigma-Aldrich, \#Z67789224EA) and the final volume adjusted to $900 \mu \mathrm{l}$ with ice-cold G-buffer. Actin was polymerized by addition of $100 \mu 110 \times$ MEK solution $\left[20 \mathrm{mM} \mathrm{MgCl}_{2}\right.$, $50 \mathrm{mM}$ glycol-bis(2-aminoethylether)- $N, N, N^{\prime}, N^{\prime}$-tetraacetic (EGTA) and $1 \mathrm{M} \mathrm{KCl}$ ] for $1 \mathrm{~h}$ at room temperature. F-actin was pelleted by ultracentrifugation for $1 \mathrm{~h}$ at room temperature at 45,000 rpm (Beckman TLA-55 rotor) and re-suspended in ice cold G-buffer. F-actin was depolymerized by dialysis against $11 \mathrm{G}$-buffer at $4^{\circ} \mathrm{C}$ for 2 days. Dialysis buffer was exchanged every morning and evening (a total of three times) Any remaining $\mathrm{F}$-actin was pelleted by ultracentrifugation at room temperature at 45,000 rpm for $30 \mathrm{~min}$ (Beckman TLA-55 rotor). Then, actin in the supernatant was loaded into HiPrep 16/60 Sephacryl S-100 HR (GE Healthcare, \#17116501) pre-equilibrated with G-buffer for a sizeexclusion chromatography (SEC). The concentration of actin was determined by measuring the absorbance at $290 \mathrm{~nm}\left[A_{290}=0.63(/ \mathrm{mg} / \mathrm{ml} /\right.$ $\mathrm{cm}$ )] using a NanoDrop 2000c spectrophotometer (Thermo Fisher Scientific).

\section{S. cerevisiae Act1 labelling with Alexa Fluor 488-maleimide or biotin-PEG2-maleimide}

S. cerevisiae Act1 was expressed and purified as described above until the digestion with chymotrypsin. After the digestion and inactivation of chymotrypsin by PMSF treatment, the eluate was collected and actin retained on the resin was also eluted with G-buffer without DTT. All the eluates were pooled in a tube and concentrated to $2.5 \mathrm{ml}$ using a $30 \mathrm{kDa}$ cutoff membrane (Sigma-Aldrich, \#Z677892-24EA). The buffer was exchanged using a PD-10 desalting column with Sephadex G-25 resin (GE-Healthcare, \#17085101) pre-equilibrated with G-buffer without DTT. The concentration of actin was adjusted to $\sim 25 \mu \mathrm{M}$ and polymerized by addition of $100 \mathrm{mM} \mathrm{KCl}$ and $2 \mathrm{mM} \mathrm{MgCl} 2$. After $1 \mathrm{~h}$ at $4{ }^{\circ} \mathrm{C}$, actin was incubated with 3-5 molar excess amount of Alexa Fluor 488 C5 maleimide (Invitrogen, \#A10254) or EZ-Link Maleimide-PEG2-Biotin (Invitrogen, \#A39261) for $1 \mathrm{~h}$ at room temperature. The cysteine-maleimide reaction was quenched by addition of $10 \mathrm{mM}$ DTT to the sample and F-actin was pelleted and re-suspended in G-buffer as described above. Monomeric G-actin was obtained by depolymerization and SEC as described above. The concentration of Alexa Fluor 488-labelled actin was measured by determining the absorbance at $290 \mathrm{~nm}$ (actin) and $495 \mathrm{~nm}\left(A_{495}\right.$; Alexa Fluor 488) on a NanoDrop 2000c spectrophotometer (Thermo Fisher Scientific), and calculated using the following formula: actin concentration $(\mathrm{M})=A_{290} /(0.63 \times 42,000)$. The extinction coefficient of Alexa Fluor 488 at $495 \mathrm{~nm}$ is $72,000 \mathrm{M}^{-1} \mathrm{~cm}^{-1}$, with a correction factor for Alexa Fluor 488 absorbance at $290 \mathrm{~nm}$ of 0.138 . Labelling efficiency was calculated as follows: $\left(A_{495} / 72,000\right) /$ actin concentration $(\mathrm{M})$. The concentration of biotin-PEG2-labelled actin was calculated by SDS-PAGE and Coomassie Brilliant Blue (CBB) staining using purified actin as the standard.

\section{Cover glass preparation for TIRF microscopy}

A microscope cover glass $(24 \times 60 \mathrm{~mm})$ was cleaned with $2 \%$ Helmanex II (Hellma, \#9-307-011-4507) for $1 \mathrm{~h}$, followed by $3 \mathrm{M} \mathrm{NaOH}$ for $30 \mathrm{~min}$ in a $60^{\circ} \mathrm{C}$ water bath sonicator. Cleaned cover glass was rinsed with pure water and dried with nitrogen gas. The cover glass was adhering to sticky-Slide VI 0.4 (ibidi, \#80608) to make six-well flow chambers. The chamber was passivated with the PLL-g-PEG and PEG-biotin mix [4 mg/ml PLL-g-PEG and $0.05 \mathrm{mg} / \mathrm{ml}$ PLL-PEG-biotin (20\%)] for $30 \mathrm{~min}$ at room temperature. The chamber was washed with G-buffer without ATP and DTT (G-AD buffer) prior to blocking with $1 \% \mathrm{BSA}$ in $\mathrm{G}-\mathrm{AD}$ buffer. The chamber was then washed with G-AD buffer prior to incubation with $0.1 \mathrm{mg} / \mathrm{ml}$ avidin in $\mathrm{G}-\mathrm{AD}$ buffer for $15 \mathrm{~min}$ at room temperature. The chamber was washed with G-AD buffer, followed by TIRF buffer [10 mM imidazole $\mathrm{pH} 7.4,50 \mathrm{mM}$ $\mathrm{KCl}, 1 \mathrm{mM} \mathrm{MgCl}$, 1 mM EGTA, $0.2 \mathrm{mM} \mathrm{ATP} \mathrm{(pH} \mathrm{7),} 10 \mathrm{mM}$ DTT, $0.5 \%$ methylcellulose (4000 cP) and $15 \mathrm{mM}$ glucose]

\section{Actin polymerization assay with TIRF microscopy}

$20 \mu \mathrm{M}$ actin consists of $\sim 80 \%$ target actin, $20 \%$ Alexa Fluor 488 -conjugated Act1 and $0.25 \%$ biotin-PEG2-Act1 were ultracentrifuged at $\sim 25$ psi with Airfuge for $10 \mathrm{~min}$ at room temperature (Beckman A-110 rotor at $160,000 \mathrm{~g}$ ). The top $75 \%$ (supernatant) fraction was collected as the sample. Actin was diluted to $1.5 \mu \mathrm{M}$ in TIRF buffer containing $12 \mathrm{kU} / \mathrm{ml}$ catalase and $48 \mathrm{kU} / \mathrm{ml}$ glucose oxidase to start its polymerization.

\section{TIRF microscopy settings and image acquisition}

For total internal reflection fluorescence (TIRF) microscopy, an Andor Revolution TIRF system was equipped with the inverted Nikon Eclipse 
microscope base, Nikon Apo 100×/1.49 NA Apo TIRF objective lens, Nikon TIRF module, a $60 \mathrm{~mW} 488 \mathrm{~nm}$ solid state laser and an Andor Zyla sCMOS camera. Images were acquired at $65 \mathrm{~nm} /$ pixel. The TIRF microscopy was performed at room temperature. Images were acquired with a $2 \mathrm{~s}$ interval.

\section{Data analysis}

The acquired images were analysed with the open source software ImageJ. Individual $\mathrm{F}$-actin filaments were traced by line segment tools and their kymographs were generated with the Kymograph builder plug-in. Actin elongation rate was calculated from the angle of the kymograph slopes and the data was processed and analysed by Python3 libraries pandas and numpy. The graph for Figs 4D and 5E were generated with the Python3 library seaborn and matplotlib, respectively. Statistical analysis for Fig. 4D was performed by using the Python3 library SciPy.

\section{Mass spectrometric analysis}

For MS, protein was reduced using $10 \mathrm{mM}$ DTT (\#MB1015, Melford) for $60 \mathrm{~min}$ at room temperature. The sample was then alkylated with $55 \mathrm{mM}$ iodoacetamide (\#I6709, Sigma) for $20 \mathrm{~min}$ in the dark at room temperature and digested using $1 \mu \mathrm{g}$ trypsin (sequencing grade; \#V5111, Promega) per $100 \mu \mathrm{g}$ of protein overnight at $37^{\circ} \mathrm{C}$. Then, $20 \mu \mathrm{l}$ samples were analysed by nano-liquid chromatography-electrospray ionization-tandem MS (LC-ESIMS/MS) using UltiMate ${ }^{\circledR} 3000$ HPLC series using the Nano Series ${ }^{\mathrm{TM}}$ Standard Columns for separation. A linear gradient from $4 \%$ to $25 \%$ solvent B $(0.1 \%$ formic acid in acetonitrile) was applied over $30 \mathrm{~min}$, followed by a step change $25-35 \%$ solvent B for $10 \mathrm{~min}$, followed by a $3 \mathrm{~min}$ wash of $90 \%$ solvent B. Peptides were directly eluted $(\sim 250 \mathrm{nl} / \mathrm{min})$ via a Triversa Nanomate nanospray source into a Orbitrap Fusion mass spectrometer (Thermo Scientific). Positive ion survey scans of peptide precursors from 375 to $1575 \mathrm{~m} / \mathrm{z}$ were performed at $120 \mathrm{~K}$ resolution (at $200 \mathrm{~m} / \mathrm{z}$ ) with automatic gain control $2 \times 105$. Precursor ions with charge state $2-6$ were isolated and subjected to higher-energy collisional dissociation (HCD) fragmentation in the IonTrap at $120 \mathrm{~K}$. MS/MS analysis was performed using collision energy of $33 \%$, automatic gain control $5 \times 103$ and $\max$ injection time of $150 \mathrm{~ms}$. The dynamic exclusion duration was set to $25 \mathrm{~s}$ with a $10 \mathrm{ppm}$ tolerance for the selected precursor and its isotopes. Monoisotopic precursor selection was turned on. Label-free quantification (LFQ) of modified precursor ions and unmodified peptides was performed using MaxQuant (v1.5.5.1). The LFQ module calculates the integrated peak area of the precursor ion based on a retention time window of $5 \mathrm{~min}$ and $\mathrm{ppm}$ error window of $10 \mathrm{ppm}$ to quantify the presence and absence of a precursor ion of interest in different MS output files. LFQ intensity data for each sample were normalized to top three unmodified actin peptides. Data was analysed in triplicate

\section{Acknowledgements}

We wish to thank members of the Balasubramanian laboratory, especially Dr Saravanan Palani and Dr Luke Springall for discussion and critical comments. We thank Dr Suresh Subramani for $P$. pastoris plasmids and strains. We thank Dr Rob Cross, who coined the Pick-ya actin acronym. We thank the Warwick Proteomics RTP for mass spectrometry.

\section{Competing interests}

The authors declare no competing or financial interests.

\section{Author contributions}

Conceptualization: T.H., M.K.B.; Methodology: T.H., L.S., A.S., M.K.B.; Validation: T.H., L.S., A.S., H.H.; Formal analysis: T.H., L.S., A.S., H.H.; Investigation: T.H., L.S., H.H.; Resources: T.H., L.S.; Data curation: T.H., H.H.; Writing - original draft: T.H., M.K.B.; Writing - review \& editing: T.H., L.S., A.S., H.H., M.K.B.;
Visualization: T.H.; Supervision: T.H., M.K.B.; Project administration: T.H., M.K.B.; Funding acquisition: M.K.B.

\section{Funding}

This work was supported by Wellcome Trust Senior Investigator Award (WT101885MA), a Wellcome Trust Collaborative Award in Science (203276/Z/16/Z), a Royal Society Wolfson merit award (WM130042) and a European Research

Council Advanced Grant (ERC-2014-ADG No. 671083), and a collaborative BBSRC award to MKB (BB/S003789/1). Deposited in PMC for immediate release.

\section{Supplementary information}

Supplementary information available online at

http://jcs.biologists.org/lookup/doi/10.1242/jcs.241406.supplemental

\section{Peer review history}

The peer review history is available online at

https://jcs.biologists.org/lookup/doi/10.1242/jcs.241406.reviewer-comments.pdf

\section{References}

Bienvenut, W. V., Sumpton, D., Martinez, A., Lilla, S., Espagne, C., Meinnel, T. and Giglione, C. (2012). Comparative large scale characterization of plant versus mammal proteins reveals similar and idiosyncratic $\mathrm{N}$ - $\alpha$-acetylation features. $\mathrm{Mol}$. Cell. Proteomics 11, M111.015131. doi:10.1074/mcp.M111.015131

Drazic, A., Aksnes, H., Marie, M., Boczkowska, M., Varland, S., Timmerman, E., Foyn, H., Glomnes, N., Rebowski, G., Impens, F. et al. (2018). NAA80 is actin's Nterminal acetyltransferase and regulates cytoskeleton assembly and cell motility. Proc. Natl. Acad. Sci. USA 17, 4399-4404. doi:10.1073/pnas.1718336115

Goris, M., Magin, R. S., Foyn, H., Myklebust, L. M., Varland, S., Ree, R., Drazic, A., Bhambra, P., Støve, S. I., Baumann, M. et al. (2018). Structural determinants and cellular environment define processed actin as the sole substrate of the N-terminal acetyltransferase NAA80. Proc. Natl. Acad. Sci. USA 17, 4405-4410. doi:10.1073/pnas.1719251115

Hatano, T., Alioto, S., Roscioli, E., Palani, S., Clarke, S. T., Kamnev, A., Hernandez-Fernaud, J. R., Sivashanmugam, L., Chapa-Y-Lazo, B., Jones A. M. E. et al. (2018). Rapid production of pure recombinant actin isoforms in Pichia pastoris. J. Cell Sci. 131, jcs213827. doi:10.1242/jcs.213827

Huang, S., Umemoto, R., Tamura, Y., Kofuku, Y., Uyeda, T. Q. P., Nishida, N. and Shimada, I. (2016). Utilization of paramagnetic relaxation enhancements for structural analysis of actin-binding proteins in complex with actin. Sci. Rep. 6, 33690. doi:10.1038/srep33690

Kalhor, H. R., Niewmierzycka, A., Faull, K. F., Yao, X., Grade, S., Clarke, S. and Rubenstein, P. A. (1999). A highly conserved 3-methylhistidine modification is absent in yeast actin. Arch. Biochem. Biophys. 370, 105-111. doi:10.1006/abbi.1999.1370

Karakozova, M., Kozak, M., Wong, C. C., Bailey, A. O., Yates, J. R., Mogilner, A., Zebroski, H. and Kashina, A. (2006). Arginylation of beta-actin regulates actin cytoskeleton and cell motility. Science 313,192-196. doi:10.1126/science.1129344

Kijima, S. T., Hirose, K., Kong, S.-G., Wada, M. and Uyeda, T. Q. P. (2016). Distinct biochemical properties of arabidopsis thaliana actin isoforms. Plant Cell Physiol. 57, 46-56. doi:10.1093/pcp/pcv176

Kwiatkowski, S., Seliga, A. K., Vertommen, D., Terreri, M., Ishikawa, T., Grabowska, I., Tiebe, M., Teleman, A. A., Jagielski, A. K., Veiga-da-Cunha, M. et al. (2018). SETD3 protein is the actin-specific histidine N-methyltransferase. Elife 7, e37921. doi:10.7554/eLife.37921

Müller, M., Diensthuber, R. P., Chizhov, I., Claus, P., Heissler, S. M., Preller, M., Taft, M. H. and Manstein, D. J. (2013). Distinct functional interactions between actin isoforms and nonsarcomeric myosins. PLOS ONE 8, e70636. doi:10.1371/ journal.pone.0070636

Noguchi, T. Q. P., Kanzaki, N., Ueno, H., Hirose, K. and Uyeda, T. Q. P. (2007). A novel system for expressing toxic actin mutants in Dictyostelium and purification and characterization of a dominant lethal yeast actin mutant. J. Biol. Chem. 282, 27721-27727. doi:10.1074/jbc.M703165200

Ohki, T., Ohno, C., Oyama, K., Mikhailenko, S. V. and Ishiwata, S. (2009) Purification of cytoplasmic actin by affinity chromatography using the C-terminal half of gelsolin. Biochem. Biophys. Res. Commun. 383, 146-150. doi:10.1016/j. bbrc.2009.03.144

Wilkinson, A. W., Diep, J., Dai, S., Liu, S., Ooi, Y. S., Song, D., Li, T.-M., Horton, J. R., Zhang, X., Liu, C. et al. (2019). SETD3 is an actin histidine methyltransferase that prevents primary dystocia. Nature 565, 372-376. doi:10. 1038/s41586-018-0821-8 